P. Cornacchia

Nagoya Math. J.

Vol. 162 (2001), 1-18

\title{
THE 2-IDEAL CLASS GROUPS OF $\mathbb{Q}\left(\zeta_{l}\right)$
}

\author{
PIETRO CORNACCHIA
}

\begin{abstract}
For prime $l$ we study the structure of the 2-part of the ideal class group $\mathrm{Cl}$ of $\mathbb{Q}\left(\zeta_{l}\right)$. We prove that $\mathrm{Cl} \otimes \mathbb{Z}_{2}$ is a cyclic Galois module for all $l<10000$ with one exception and compute the explicit structure in several cases.
\end{abstract}

\section{$\S 1$. Introduction}

Let $l$ be an odd prime number and let $\zeta_{l}$ be a primitive $l$-th root of unity. We denote by $\mathrm{Cl}$ the ideal class group of the field $\mathbb{Q}\left(\zeta_{l}\right)$. The aim of this paper is to study the structure of the 2-part of $\mathrm{Cl}$ as an abelian group. Let $G$ be the Galois group $\operatorname{Gal}\left(\mathbb{Q}\left(\zeta_{l}\right) / \mathbb{Q}\right)$. We have a natural decomposition $G=\Delta \times P$ where $P$ is the 2-Sylow subgroup of $G$ and $\Delta$ is the subgroup of $G$ consisting of the elements of odd order. Let $\mathrm{Cl}^{+}$be the ideal class group of the field $\mathbb{Q}\left(\zeta_{l}+\zeta_{l}^{-1}\right)$; there exists a natural injective map $\mathrm{Cl}^{+} \rightarrow \mathrm{Cl}$, and we denote by $\mathrm{Cl}^{-}$its cokernel. In order to study the 2-part of class groups, it is useful to introduce 2-adic characters. Let $\chi: \Delta \rightarrow \overline{\mathbb{Q}}_{2}^{*}$ be a 2-adic character, and denote by $\mathcal{O}_{\chi}$ the ring $\mathbb{Z}_{2}(\chi)$. For any $\mathbb{Z}[G]$-module $M$, we define its $\chi$-part $M(\chi)$ as $\left(M \otimes_{\mathbb{Z}} \mathbb{Z}_{2}\right) \otimes_{\mathbb{Z}_{2}[\Delta]} \mathcal{O}_{\chi}$. It is an $\mathcal{O}_{\chi}[P]$-module. In particular, the 2-part $M \otimes_{\mathbb{Z}} \mathbb{Z}_{2}$ of $M$ is a direct sum of $\chi$-parts. For more information on $\chi$-parts, see [4]. In Section 2 we prove a cyclicity criterion, which is a version of théorème I.9 of [10] adapted to our situation:

Theorem $1 . \quad$ Let $l \equiv 1(\bmod 4)$. The group $\mathrm{Cl}(\chi)$ is a nontrivial cyclic $\mathcal{O}_{\chi}$-module if and only if $\# \mathrm{Cl}^{-}(\chi)=\#\left(\mathcal{O}_{\chi} / 2\right)$.

The above theorem allows one to determine $\mathrm{Cl}(\chi)$ in some cases. As an example, consider the field $\mathbb{Q}\left(\zeta_{9337}\right)$. Let $\chi$ be the character of order 3 . We have $\# \mathrm{Cl}^{-}(\chi)=\#\left(\mathcal{O}_{\chi} / 2\right)$, and $\# \mathrm{Cl}^{+}(\chi)=\#\left(\mathcal{O}_{\chi} / 8\right)$. Applying the theorem, we get $\mathrm{Cl}(\chi) \cong \mathcal{O}_{\chi} / 16$.

Received March 10, 1998.

1991 Mathematics Subject Classification: 11R29, 11R18, 11R27.

The present study was partially supported by a CNR grant. 
We then characterize the cohomological triviality of the $\mathcal{O}_{\chi}[P]$-module $\mathrm{Cl}(\chi)$. In Proposition 4 we show that $\mathrm{Cl}(\chi)$ is cohomologically trivial if and only if the $\chi$-part of the units of $\mathbb{Q}\left(\zeta_{l}+\zeta_{l}^{-1}\right)$ have independent signs.

In Section 3 we study the cyclicity of $\mathrm{Cl}(\chi)$ as an $\mathcal{O}_{\chi}[P]$-module. If $\mathrm{Cl}(\chi)$ is cyclic, then it is possible to compute explicitly the $\mathcal{O}_{\chi}[P]$-structure of $\mathrm{Cl}^{+}(\chi)$ and of $\mathrm{Cl}^{-}(\chi)$. The structure of $\mathrm{Cl}^{-}(\chi)$ is given by Proposition 2. The description of the structure of $\mathrm{Cl}^{+}(\chi)$ is more complicated. There exists an ideal $J^{+}(\chi)$ of $\mathcal{O}_{\chi}[P]$ such that $\mathrm{Cl}^{+}(\chi)$ and $\mathcal{O}_{\chi}[P] / J^{+}(\chi)$ have the same order. The definition of $J^{+}(\chi)$ can be found in Proposition 9 of [4]. The ideal $J^{+}(\chi)$ annihilates $\mathrm{Cl}^{+}(\chi)$ (Theorem 2.2 of [13]): this is proved using methods developed by $\mathrm{F}$. Thaine. A more precise result is also given in [5]. Therefore, in case $\mathrm{Cl}^{+}(\chi)$ is cyclic over $\mathcal{O}_{\chi}[P]$, we have $\mathrm{Cl}^{+}(\chi) \cong \mathcal{O}_{\chi}[P] / J^{+}(\chi)$. The ideals $J^{+}(\chi)$ have been computed in [17] for all fields of prime conductor $l<10000$. Cyclicity questions have been studied in [15], where it is proved that the minus class group $\mathrm{Cl}^{-}$of $\mathbb{Q}\left(\zeta_{l}\right)$ is a cyclic Galois module for all primes $l \leq 509$. For class groups of real cyclic fields there are also some results in this direction [1, 7]. Numerical computations suggest that $\mathrm{Cl}(\chi)$ is a cyclic $\mathcal{O}_{\chi}[P]$-module most of the times, and it is quite hard to find examples when this condition is not verified. We prove that $\mathrm{Cl}(\chi)$ is cyclic whenever $\mathrm{Cl}^{+}(\chi)$ is trivial (Propositions 5 and 6). Moreover, we prove the following:

THEOREM 2. If $l<10000$ is a prime number not equal to 7687 , then the group $\mathrm{Cl}(\chi)$ is a cyclic Galois module.

In the case $l=7687$ and $\chi$ a nontrivial cubic character, one could show by explicit computations that $\mathrm{Cl}(\chi)$ has actually two generators. In Section 4 we give several structure results on the $\mathcal{O}_{\chi}$-structure of $\mathrm{Cl}(\chi)$ in the case that $\mathrm{Cl}(\chi)$ is a cyclic $\mathcal{O}_{\chi}[P]$-module. In particular, we determine completely the $\mathcal{O}_{\chi}$-structure of $\mathrm{Cl}(\chi)$ when $l \equiv 3(\bmod 4), \mathrm{Cl}(\chi)$ is a cyclic $\mathcal{O}_{\chi}[P]$-module and $\# \mathrm{Cl}^{+}(\chi) \neq \# \mathrm{Cl}^{-}(\chi)$ (Propositions 7 and 8). These results allow us to determine in many cases the structure of $\mathrm{Cl}(\chi)$ as an $\mathcal{O}_{\chi^{-}}$ module. The numerical results are presented in the table which is described in Section 5.

\section{$\S 2$. Generalities on class groups}

We maintain the same notations as in the introduction. Let $G$ be the Galois group of the field $\mathbb{Q}\left(\zeta_{l}\right)$ over the rationals. The group $G$ is a cyclic abelian group of order $l-1$. Let $2^{e}$ be the exact power of 2 dividing $l-1$. 
Let $\chi: \Delta \rightarrow \overline{\mathbb{Q}}_{2}^{*}$ be a 2-adic character of $\Delta$, and denote by $\mathcal{O}_{\chi}$ the discrete valuation ring $\mathbb{Z}_{2}(\chi)$. Let $\mathrm{Cl}(\chi)$ be the $\chi$-part of the ideal class group of $\mathbb{Q}\left(\zeta_{l}\right)$. For any $\mathbb{Z}[G]$-module $M$, we define its $\chi$-part

$$
M(\chi)=\left(M \otimes_{\mathbb{Z}} \mathbb{Z}_{2}\right) \otimes_{\mathbb{Z}_{2}[\Delta]} \mathcal{O}_{\chi}
$$

We are interested in the structure of $\mathrm{Cl}(\chi)$ as a $\mathcal{O}_{\chi}[P]$-module. In order to proceed, we introduce some notation. Let $d$ be the order of the character $\chi$. We denote by $K_{e}$ the subfield of $\mathbb{Q}\left(\zeta_{l}\right)$ fixed by Ker $\chi$. It is a cyclic extension of $\mathbb{Q}$ of degree $d \cdot 2^{e}$. For all $0 \leq i \leq e$, we denote by $K_{i}$ the unique subfield of $K_{e}$ of degree $d \cdot 2^{i}$ over $\mathbb{Q}$. The fields $K_{i}$ are totally real abelian fields for all $0 \leq i \leq e-1$. We denote by $\mathrm{Cl}_{i}$ and by $\mathrm{Cl}_{i}^{\infty}$ the ideal class group and the narrow ideal class group respectively of the field $K_{i}$. We also write $\mathrm{Cl}_{e}^{+}$ for $\mathrm{Cl}_{e-1}$. Observe that $\mathrm{Cl}_{e} \cong \mathrm{Cl}_{e}^{\infty}$.

Proposition 1. Let the notations be as above. For $i \geq j$, we denote by $\sigma_{i, j}$ a generator of $\operatorname{Gal}\left(K_{i} / K_{j}\right)$. Then we have

$$
\begin{gathered}
\mathrm{Cl}_{i}^{\infty} \cong \mathrm{Cl}_{e} /\left(\mathrm{Cl}_{e}\right)^{1-\sigma_{e, i}}, \forall 0 \leq i \leq e \\
\mathrm{Cl}_{i} \cong \mathrm{Cl}_{e-1} /\left(\mathrm{Cl}_{e-1}\right)^{1-\sigma_{e-1, i}}, \forall 0 \leq i \leq e-1
\end{gathered}
$$

Proof. The proof is the same as the one of Lemma 1 of [3]. We prove (1); the proof of (2) is analogous. The extension $K_{e} / K_{i}$ is totally ramified at the unique prime ideal of $K_{i}$ above $l$ and unramified above all other finite primes. Class field theory implies that the norm map $N_{e, i}: \mathrm{Cl}_{e} \rightarrow \mathrm{Cl}_{i}^{\infty}$ is surjective. The group $\left(\mathrm{Cl}_{e}\right)^{1-\sigma_{e, i}}$ is clearly contained in the kernel. Applying the genus theory formula (see [11], Chapter 13, Lemma 4.1) and Hasse's principle, it follows that $\# \mathrm{Cl}_{i}^{\infty}=\# \mathrm{Cl}_{e}^{\mathrm{Gal}\left(K_{e} / K_{i}\right)}=\# \mathrm{Cl}_{e} /\left(\mathrm{Cl}_{e}\right)^{1-\sigma_{e, i}}$. Therefore the map $N_{e, i}$ induces an isomorphism, and our claim is proved.

We define the minus class group $\mathrm{Cl}_{e}^{-}$to be the cokernel of the natural $\operatorname{map} \mathrm{Cl}_{e-1} \rightarrow \mathrm{Cl}_{e}$.

Let $j \in G$ denote complex conjugation. Let $\zeta_{2^{e}} \in \overline{\mathbb{Q}}_{2}$ be a primitive $2^{e}$-th root of unity. There exists an isomorphism $\mathrm{Cl}_{e}^{-}(\chi) \cong \mathrm{Cl}^{-}(\chi)$ induced by the norm map from $\mathbb{Q}\left(\zeta_{l}\right)$ to $K_{e}$. The group $\mathrm{Cl}^{-}(\chi)$ is an $\mathcal{O}_{\chi}[P] /(1+$ $j) \cong \mathcal{O}_{\chi}\left[\zeta_{2^{e}}\right]$-module. Suppose that $\mathrm{Cl}^{-}(\chi)$ is a cyclic Galois module. Since $\mathcal{O}_{\chi}\left[\zeta_{2^{e}}\right]$ is a discrete valuation ring, there is a simple description of the structure of $\mathrm{Cl}^{-}(\chi)$. Let $2^{f}=\#\left(\mathcal{O}_{\chi} / 2\right)$. The following is Proposition 3.4 of $[15]$. 
Proposition 2. Suppose that $A$ is a cyclic $\mathcal{O}_{\chi}[P] /(1+j)$-module. Moreover suppose that \#A=2 $2^{f t}$. Then there is an isomorphism of $\mathcal{O}_{\chi}\left[\zeta_{2^{e}}\right]$ modules

$$
A \cong \mathcal{O}_{\chi}\left[\zeta_{2^{e}}\right] /\left(1-\zeta_{2^{e}}\right)^{t}
$$

and an isomorphism of $\mathcal{O}_{\chi}$-modules

$$
A \cong\left(\mathcal{O}_{\chi} / 2^{r}\right)^{\left(2^{e-1}-s\right)} \times\left(\mathcal{O}_{\chi} / 2^{r+1}\right)^{s}
$$

where $r, s \in \mathbb{N}$ are determined by $t=r 2^{e-1}+s$ and $0 \leq s<2^{e-1}$.

Proof. This follows because $\mathcal{O}_{\chi}\left[\zeta_{2^{e}}\right]$ is a discrete valuation ring with uniformizing element $1-\zeta_{2}$.

For any $\mathcal{O}_{\chi}$-module $A$, we denote by $\operatorname{rank}_{\mathcal{O}_{\chi}} A$ the dimension of the $\mathcal{O}_{\chi} / 2$-vector space $A / 2$.

Corollary 1. Let $\# \mathrm{Cl}^{-}(\chi)=2^{\text {ft }}$ and suppose that $\mathrm{Cl}^{-}(\chi)$ is a cyclic $\mathcal{O}_{\chi}[P]$-module. Then $\operatorname{rank}_{\mathcal{O}_{\chi}} \mathrm{Cl}^{-}(\chi)=\min \left(t, 2^{e-1}\right)$.

Proof. Since $1+j$ annihilates $\mathrm{Cl}^{-}(\chi)$, we can apply Proposition 2 with $A=\mathrm{Cl}^{-}(\chi)$. If $t<2^{e-1}$, then $r=0, s=t$ and (4) gives us $\operatorname{rank}_{\mathcal{O}_{\chi}} \mathrm{Cl}^{-}(\chi)=$ $s=t$. If $t \geq 2^{e-1}$ then $r>0$ and (4) gives us $\operatorname{rank}_{\mathcal{O}_{\chi}} \mathrm{Cl}^{-}(\chi)=2^{e-1}$, as we wanted to show.

We are now ready to prove Theorem 1 of the introduction.

Proof of Theorem 1. Suppose that $\mathrm{Cl}(\chi) \cong \mathrm{Cl}_{e}(\chi)$ is nontrivial and cyclic over $\mathcal{O}_{\chi}$. This implies that $\mathrm{Cl}^{-}(\chi)$ is a nontrivial cyclic $\mathcal{O}_{\chi}$-module. The condition on $l$ is equivalent to say that $e>1$. Corollary 1 implies $t=1$, therefore $\# \mathrm{Cl}^{-}(\chi)=2^{f}=\#\left(\mathcal{O}_{\chi} / 2\right)$. Suppose now that $\# \mathrm{Cl}^{-}(\chi)=$ $\#\left(\mathcal{O}_{\chi} / 2\right)$. In particular, $\mathrm{Cl}^{-}(\chi)$ is a cyclic $\mathcal{O}_{\chi}[P]$-module. We have $\mathrm{Cl}^{-}(\chi) \cong$ $\mathrm{Cl}(\chi) / \mathrm{Cl}(\chi)^{1+j}$ and $1+j$ is in the maximal ideal of $\mathcal{O}_{\chi}[P]$. Nakayama's lemma implies that the group $\mathrm{Cl}(\chi)$ is a cyclic $\mathcal{O}_{\chi}[P]$-module. We identify the ring $\mathcal{O}_{\chi}[P]$ with the ring $R=\mathcal{O}_{\chi}[T] /\left((1+T)^{2^{e}}-1\right)$. Since $R / 2 \cong$ $\frac{\mathcal{O}_{\chi}}{2}[T] /\left(T^{2^{e}}\right)$, we have $\mathrm{Cl}(\chi) / 2 \cong \frac{\mathcal{O}_{\chi}}{2}[T] /\left(T^{h}\right)$ for some $h \leq 2^{e}$. Since $1+j=$ $1+(1+T)^{2^{e-1}}$, we obtain $\mathrm{Cl}^{-}(\chi) / 2 \cong \frac{\mathcal{O}_{\chi}}{2}[T] /\left(T^{h}, T^{2^{e-1}}\right)$. Our assumption implies that $\min \left(h, 2^{e-1}\right)=1$. Since $e>1$, we must have $h=1$ : this means that $\mathrm{Cl}(\chi)$ is a cyclic $\mathcal{O}_{\chi}$-module. 
We now study the cohomology of the groups $\mathrm{Cl}(\chi)$. We say that $\mathrm{Cl}(\chi)$ is cohomologically trivial if the Tate cohomology groups $\widehat{H}^{i}(P, \mathrm{Cl}(\chi))$ are trivial for all $i \in \mathbb{Z}$. Since $P$ is a cyclic group and $\mathrm{Cl}(\chi)$ has finite order, Tate cohomology has period 2 and the Herbrand quotient is 1 . Therefore saying that $\mathrm{Cl}(\chi)$ is cohomologically trivial is equivalent to say that there exists an $i$ such that $\widehat{H}^{i}(P, \mathrm{Cl}(\chi))$ is trivial.

We need to recall some notations and results. For any field $E$, we denote by $\mathcal{O}_{E}^{*}$ the unit group of its ring of integers. If $E$ is a totally real field, we also denote by $E_{+}$the set of totally positive elements of $E$, and by $\mathcal{O}_{E,+}^{*}$ the group of totally positive units in $\mathcal{O}_{E}^{*}$. Combining Theorem 1 of [4] and Proposition 7 (iii) of [4] we get that

$$
\widehat{H}^{0}(P, \mathrm{Cl}(\chi)) \cong\left(\mathcal{O}_{K_{0},+}^{*} / N_{K_{0}}^{K_{e}} \mathcal{O}_{K_{e}}^{*}\right)(\chi)
$$

where $N_{K_{0}}^{K_{e}}$ is the norm map from $K_{e}$ to $K_{0}$. We need to recall another result.

Proposition 3. Let $K$ be a totally real number field and let $K / E$ be a quadratic extension. Suppose that $\left(\mathcal{O}_{E}^{*}\right)^{2}=\mathcal{O}_{E,+}^{*}$. We then have a natural isomorphism

$$
K^{*} /\left(K_{+}^{*} \mathcal{O}_{K}^{*}\right) \cong \widehat{H}^{0}\left(\operatorname{Gal}(K / E), \mathcal{O}_{K}^{*}\right) .
$$

For a proof see [2], Theorem 12.11, page 61. We now give a criterion for the cohomological triviality of $\mathrm{Cl}(\chi)$ in terms of the signature of the units.

Proposition 4. The cohomology group $\widehat{H}^{0}(P, \mathrm{Cl}(\chi))$ is trivial if and only if $\left(\mathcal{O}_{K_{e-1},+}^{*} /\left(\mathcal{O}_{K_{e-1}}^{*}\right)^{2}\right)(\chi) \cong 0$.

Proof. Since $\mathcal{O}_{K_{e}}^{*}=\mu\left(K_{e}\right) \mathcal{O}_{K_{e-1}}^{*}$, where $\mu\left(K_{e}\right)$ are the roots of unity in $K_{e}$ (the Hasse index is 1 in our situation), $N_{K_{0}}^{K_{e}} \mathcal{O}_{K_{e}}^{*}=\left(N_{K_{0}}^{K_{e-1}} \mathcal{O}_{K_{e-1}}^{*}\right)^{2}$. We have

$$
\left(N_{K_{0}}^{K_{e-1}} \mathcal{O}_{K_{e-1}}^{*}\right)^{2} \subset\left(\mathcal{O}_{K_{0}}^{*}\right)^{2} \subset \mathcal{O}_{K_{0},+}^{*}
$$

Therefore, by (5), $\widehat{H}^{0}(P, \mathrm{Cl}(\chi)) \cong 0$ is equivalent to

$$
\left(N_{K_{0}}^{K_{e-1}} \mathcal{O}_{K_{e-1}}^{*}\right)(\chi)=\mathcal{O}_{K_{0}}^{*}(\chi) \text { and }\left(\mathcal{O}_{K_{0}}^{*}\right)^{2}(\chi)=\mathcal{O}_{K_{0},+}^{*}(\chi) .
$$

If $e=1$, then $K_{e-1}=K_{0}$ and we are done. From now on, we suppose that $e>1$. Since the isomorphism in Proposition 3 is natural, it remains true if 
we take $\chi$-parts. Suppose that (6) holds. The first condition, which can be stated as ${ }^{1}$

$$
\widehat{H}^{0}\left(\operatorname{Gal}\left(K_{e-1} / K_{0}\right), \mathcal{O}_{K_{e-1}}^{*}(\chi)\right) \cong 0
$$

implies that

$$
\widehat{H}^{0}\left(\operatorname{Gal}\left(K_{i+1} / K_{i}\right), \mathcal{O}_{K_{i+1}}^{*}(\chi)\right) \cong 0, \forall 0 \leq i \leq e-2 .
$$

We apply inductively Proposition 3 to the extensions $K_{i+1} / K_{i}$, until $i=$ $e-2$. At each step we get $\left(K_{i+1}\right)^{*} /\left(\left(K_{i+1}\right)_{+}^{*} \mathcal{O}_{K_{i+1}}^{*}\right)(\chi) \cong 0$, which is equivalent to $\left(\mathcal{O}_{K_{i+1},+}^{*} /\left(\mathcal{O}_{K_{i+1}}^{*}\right)^{2}\right)(\chi) \cong 0$. The last step gives our claim. Vice versa, suppose that the group $\left(\mathcal{O}_{K_{e-1},+}^{*} /\left(\mathcal{O}_{K_{e-1}}^{*}\right)^{2}\right)(\chi)$ is trivial. Since the extension $K_{e-1} / K_{0}$ is totally ramified above $l \neq 2$, we have that $\left(\mathcal{O}_{K_{i},+}^{*} /\left(\mathcal{O}_{K_{i}}^{*}\right)^{2}\right)(\chi)$ is trivial for all $i=1, \ldots, e-1$. Therefore using Proposition 3 again, we have that at each step the cohomology group $\widehat{H}^{0}\left(K_{i+1} / K_{i}\right.$, $\left.\mathcal{O}_{K_{i+1}}^{*}(\chi)\right)$ is trivial. This implies that $\mathcal{O}_{K_{0}}^{*}(\chi) \cong N_{K_{0}}^{K_{e-1}} \mathcal{O}_{K_{e-1}}^{*}(\chi)$, thus $(6)$ is satisfied.

\section{$\S 3$. Cyclicity of $\mathrm{Cl}(\chi)$ as a Galois module}

In this section we study the case when $\mathrm{Cl}(\chi)$ is a cyclic $\mathcal{O}_{\chi}[P]$-module. Numerical computations suggest that this is almost always the case, and in this situation we have more information on the structure of $\mathrm{Cl}(\chi)$. We maintain the notations from the previous sections. The norm map $\mathrm{Cl} \rightarrow \mathrm{Cl}^{+}$ is surjective by class field theory, and the natural map $\mathrm{Cl}^{+} \rightarrow \mathrm{Cl}$ is injective [11, Chap. 3, Th. 4.2]. The composition of these maps is multiplication by $1+j$; this allows us to identify $\mathrm{Cl}^{+}$with $\mathrm{Cl}^{1+j}$.

We first state a criterion of cyclicity of $\mathrm{Cl}_{0}(\chi)$ as a $\mathcal{O}_{\chi}$-module.

Theorem 3. (T. Berthier) Suppose that $\chi$ is not the trivial character. If $\operatorname{rank}_{\mathcal{O}_{\chi}} \mathrm{Cl}_{0}(\chi)=1$ then there exists a prime number $r \equiv 3(\bmod 4)$ which is split in $K_{0} / \mathbb{Q}$, such that the $\chi$-part ${ }^{2} \mathrm{Cl}_{\chi, r}$ of the ideal class group of the field $K_{0}(\sqrt{-r})$ has order $\# \mathrm{Cl}_{0}(\chi) \#\left(\mathcal{O}_{\chi} / 2\right)$. On the other hand, if there exists a prime $r$ as above, then $\operatorname{rank}_{\mathcal{O}_{\chi}} \mathrm{Cl}_{0}(\chi) \leq 1$.

\footnotetext{
${ }^{1}$ We remark that if $\chi$ is not the trivial character, then it is true that (7) is equivalent to say that $\mathcal{O}_{K_{e-1}}^{*}(\chi)$ is a free one dimensional $\mathcal{O}_{\chi}\left[\mathrm{Gal}\left(K_{e-1} / K_{0}\right)\right]$-module, but we do not need this.

${ }^{2}$ Here we view $\chi$ as a character of $\operatorname{Gal}\left(K_{0} / \mathbb{Q}\right)$ and extend it to
$\operatorname{Gal}\left(K_{0}(\sqrt{-r}) / \mathbb{Q}(\sqrt{-r})\right)$.
} 
This result is a special case of [1, Th. 2.4.3]. The proof of the first part is difficult. Here we sketch the proof of the second part. The conditions imposed on $r$ imply that the places which ramify in $K_{0}(\sqrt{-r}) / K_{0}$ are precisely the infinite ones and the ones above $r$. If we apply the $\chi$-ambiguous class number formula of genus theory ${ }^{3}$, we get that $\mathrm{Cl}_{\chi, r}^{\mathrm{Gal}\left(K_{0}(\sqrt{-r}) / K_{0}\right)}$ has order at least $\# \mathrm{Cl}_{0}(\chi) \#\left(\mathcal{O}_{\chi} / 2\right)$. Therefore our hypothesis force $\mathrm{Cl}_{\chi, r}$ to be $\operatorname{Gal}\left(K_{0}(\sqrt{-r}) / K_{0}\right)$-invariant. Since the field $K_{0}(\sqrt{-r})$ is totally imaginary, the field $K_{0}$ is totally real, and $\chi$ is not the trivial character, it is not hard to show that the natural map $\mathrm{Cl}_{0}(\chi) \rightarrow \mathrm{Cl}_{\chi, r}$ is injective. Moreover, since the extension $K_{0}(\sqrt{-r}) / K_{0}$ is ramified, the norm map $\mathrm{Cl}_{\chi, r} \rightarrow \mathrm{Cl}_{0}(\chi)$ is surjective. Let $\sigma$ be a generator of $\operatorname{Gal}\left(K_{0}(\sqrt{-r}) / K_{0}\right)$. We have $\mathrm{Cl}_{\chi, r}^{2}=\mathrm{Cl}_{\chi, r}^{1+\sigma} \cong \mathrm{Cl}_{0}(\chi)$. Therefore

$$
\#\left(\mathrm{Cl}_{\chi, r} / \mathrm{Cl}_{\chi, r}^{2}\right)=\#\left(\mathcal{O}_{\chi} / 2\right)
$$

and we get that $\operatorname{rank}_{\mathcal{O}_{\chi}} \mathrm{Cl}_{\chi, r}=1$. Since $\mathrm{Cl}_{0}(\chi)$ is an epimorphic image of $\mathrm{Cl}_{\chi, r}$, we get $\operatorname{rank}_{\mathcal{O}_{\chi}} \mathrm{Cl}_{0}(\chi) \leq 1$.

Corollary 2. If $\mathrm{Cl}_{0}(\chi)$ is a cyclic $\mathcal{O}_{\chi}$-module, then $\mathrm{Cl}_{0}^{\infty}(\chi)$ is a cyclic $\mathcal{O}_{\chi}$-module as well.

Proof. We can assume that $\mathrm{Cl}_{0}(\chi)$ is nontrivial, otherwise $\mathrm{Cl}_{0}^{\infty}(\chi)$ is either trivial or isomorphic to $\mathcal{O}_{\chi} / 2$, hence cyclic. In this situation $\chi$ is not the trivial character and we can apply Theorem 3 . Therefore there exists a quadratic totally imaginary extension $E=K_{0}(\sqrt{-r})$ of $K_{0}$ such that the $\chi$-part $\mathrm{Cl}_{E}(\chi)$ of the ideal class group of $E$ has $\mathcal{O}_{\chi}$-rank equal to 1 (see the proof of Theorem 3). Moreover the extension $E / K_{0}$ is ramified at the finite primes above $r$. Therefore the norm map $\mathrm{Cl}_{E}(\chi) \rightarrow \mathrm{Cl}_{0}^{\infty}(\chi)$ between narrow ideal class groups is surjective. The group $\mathrm{Cl}_{0}^{\infty}(\chi)$ is then a surjective image of $\mathrm{Cl}_{E}(\chi)$ (they are actually isomorphic). Therefore $\mathrm{Cl}_{0}^{\infty}(\chi)$ is a cyclic $\mathcal{O}_{\chi^{-}}$ module.

Observe that in the case -1 is a power of 2 modulo the order of $\chi$, B. Oriat already proved the equality $\operatorname{rank}_{\mathcal{O}_{\chi}} \mathrm{Cl}_{0}(\chi)=\operatorname{rank}_{\mathcal{O}_{\chi}} \mathrm{Cl}_{0}^{\infty}(\chi)$ using the Spiegelungssatz [12, Cor. 2 c].

Remark. In [15, Th. 3.3] a sufficient condition for $\mathrm{Cl}^{-}(\chi)$ to be a cyclic $\mathcal{O}_{\chi}[P]$-module is given.

\footnotetext{
${ }^{3}$ This is the $\chi$-version of Lemma 4.1 , Chapter 13 of [11]. See also [6].
} 
Proposition 5. The following assertions are equivalent:

1. $\mathrm{Cl}(\chi)$ is a cyclic $\mathcal{O}_{\chi}[P]$-module;

2. $\mathrm{Cl}^{-}(\chi)$ is a cyclic $\mathcal{O}_{\chi}[P]$-module;

3. $\mathrm{Cl}^{+}(\chi)$ is a cyclic $\mathcal{O}_{\chi}[P]$-module;

4. $\mathrm{Cl}_{0}^{\infty}(\chi)$ is a cyclic $\mathcal{O}_{\chi}$-module;

5. $\mathrm{Cl}_{0}(\chi)$ is a cyclic $\mathcal{O}_{\chi}$-module.

Proof. The ring $\mathcal{O}_{\chi}[P]$ is a local ring with maximal ideal $(2,1-\sigma)$, where $\sigma$ is a generator of $P$. By definition

$$
\mathrm{Cl}^{-}(\chi) \cong \mathrm{Cl}(\chi) / \mathrm{Cl}^{+}(\chi) \cong \mathrm{Cl}(\chi) / \mathrm{Cl}(\chi)^{1+j} .
$$

Nakayama's lemma gives the equivalence of 1 and 2. The equivalence of 1 and 4 follows again by Nakayama's lemma and Equation (1) of Proposition 1. Similarly, 3 and 5 are equivalent by Nakayama's lemma and Equation (2) of Proposition 1. Since $\mathrm{Cl}_{0}(\chi)$ is a surjective image of $\mathrm{Cl}_{0}^{\infty}(\chi)$, condition 4 implies 5. Moreover 5 implies 4 by Corollary 2.

Sometimes it is easy to show that $\mathrm{Cl}(\chi)$ is a cyclic Galois module.

Proposition 6. Suppose that $\mathrm{Cl}^{+}(\chi) \cong 0$. Then $\mathrm{Cl}(\chi)$ is a cyclic $\mathcal{O}_{\chi}[P]$-module.

Proof. This is immediate from Proposition 5, but we give a direct proof independent of Corollary 2. In the notation of the previous section, the group $\mathrm{Cl}_{0}(\chi)$ is trivial, because it is a surjective image of $\mathrm{Cl}_{e-1}(\chi)=\mathrm{Cl}^{+}(\chi)$ under the norm map. This implies that $\mathrm{Cl}_{0}^{\infty}(\chi)$ is either trivial, or isomorphic to $\mathcal{O}_{\chi} / 2$. Therefore in both cases $\mathrm{Cl}_{0}^{\infty}(\chi)$ is a cyclic $\mathcal{O}_{\chi}[P]$-module. By Proposition 1 we have $\mathrm{Cl}_{0}^{\infty}(\chi) \cong \mathrm{Cl}(\chi) / \mathrm{Cl}(\chi)^{1-\sigma}$. By Nakayama's lemma, we get that $\mathrm{Cl}(\chi)$ is cyclic, as we had to show.

Proof of Theorem 2. Because of Proposition 6, we can suppose that $\mathrm{Cl}^{+}(\chi)$ is not trivial. In [4] we determined all 2-adic characters $\chi$ of conductor $l<10000$ such that $\mathrm{Cl}^{+}(\chi)$ is not trivial. They also appear in the table at the end of this paper. By Proposition 5 we can rule out all cases with either $\# \mathrm{Cl}^{+}(\chi) \leq \#\left(\mathcal{O}_{\chi} / 2\right)$ or $\# \mathrm{Cl}^{-}(\chi) \leq \#\left(\mathcal{O}_{\chi} / 2\right)$. Only few cases remain; they are precisely the characters of order 3 and conductors $l=349$, 
709, 1777, 4261, 4297, 4357, 4561, 6247, 7687, 9109. For these characters, it is enough to check whether condition 5 of Proposition 5 holds. Looking at the tables in [8], one sees that for $l=349,709,4261,4357,4561,9109$, the group $\mathrm{Cl}_{0}(\chi)$ has order $4=\#\left(\mathcal{O}_{\chi} / 2\right)$, hence it is $\mathcal{O}_{\chi}$-cyclic. Since we exclude $l=7687$, to complete the proof we are left with the three cases $l=1777,4297,6247$. To prove the cyclicity of $\mathrm{Cl}_{0}(\chi)$ it is enough to find in each case an auxiliary prime $r$ satisfying the conditions of Theorem 3 . This has been done in [1]: if $l=1777$ or $l=4297$ one can take $r=7$, for $l=6247$ one takes $r=11$. The proof of the theorem is now complete.

\section{$\S 4$. Structure of $\mathrm{Cl}(\chi)$ as an $\mathcal{O}_{\chi}$-module}

Suppose we are given a prime number $l$, a character $\chi$ as in the previous sections, and we know that $\mathrm{Cl}(\chi)$ is a cyclic $\mathcal{O}_{\chi}[P]$-module. In several cases it is possible to determine the structure of $\mathrm{Cl}(\chi)$ as an $\mathcal{O}_{\chi}$-module from the knowledge of $h_{\chi}^{+}=\# \mathrm{Cl}^{+}(\chi), h_{\chi}^{-}=\# \mathrm{Cl}^{-}(\chi)$ and the order of the cohomology groups. In this section we give several criteria in this direction.

\subsection{The case $l \equiv 3(\bmod 4)$}

The case $l \equiv 3(\bmod 4)$ is simpler because $P$ is cyclic of order 2 , generated by complex conjugation $j$. Suppose that $\mathrm{Cl}(\chi)$ is a cyclic Galois module. In this situation both $\mathrm{Cl}^{+}(\chi)$ and $\mathrm{Cl}^{-}(\chi)$ are cyclic $\mathcal{O}_{\chi}$-modules. Let $f_{\chi}$ be the dimension of $\mathcal{O}_{\chi} / 2$, as a $\mathbb{Z} / 2 \mathbb{Z}$-vector space. We denote by $a_{\chi}^{+}$ and by $a_{\chi}^{-}$respectively the integers $\sqrt[f_{\chi}]{h_{\chi}^{+}}$and $\sqrt[f_{\chi}]{h_{\chi}}-$ They are defined in such a way that

$$
\#\left(\mathcal{O}_{\chi} / a_{\chi}^{+}\right)=h_{\chi}^{+} \text {and } \#\left(\mathcal{O}_{\chi} / a_{\chi}^{-}\right)=h_{\chi}^{-}
$$

Let $a_{\chi}^{\max }=\max \left(a_{\chi}^{+}, a_{\chi}^{-}\right)$and $a_{\chi}^{\min }=\min \left(a_{\chi}^{+}, a_{\chi}^{-}\right)$. $2 a_{\chi}^{\max }$.

Lemma 1. Assume that $l \equiv 3(\bmod 4)$. Then $\mathrm{Cl}(\chi)$ is annihilated by

Proof. Let $x \in \mathrm{Cl}(\chi)$. We have

$$
x^{2}=x^{1+j} x^{1-j}
$$

We have $\mathrm{Cl}(\chi)^{a_{\chi}^{+}(1+j)}=1$. Since

$$
\mathrm{Cl}(\chi)^{1-j} \subset \operatorname{Ker}\left(1+j: \mathrm{Cl}(\chi) \rightarrow \mathrm{Cl}(\chi)^{1+j}\right)
$$


we get that

$$
\# \mathrm{Cl}(\chi)^{1-j} \leq h_{\chi}^{-}
$$

therefore $\mathrm{Cl}(\chi)^{a_{\chi}^{-}(1-j)}=1$. If we apply $a_{\chi}^{\max }$ to $(8)$ we get $x^{2 a_{\chi}^{\max }}=1$, as we wanted to show.

Corollary $3 . \quad$ Let $l \equiv 3(\bmod 4)$. Suppose that $\mathrm{Cl}(\chi)$ is a nontrivial cyclic $\mathcal{O}_{\chi}[P]$-module. Then, either

$$
\mathrm{Cl}(\chi) \cong\left(\mathcal{O}_{\chi} / a_{\chi}^{+}\right) \times\left(\mathcal{O}_{\chi} / a_{\chi}^{-}\right)
$$

or

$$
\mathrm{Cl}(\chi) \cong\left(\mathcal{O}_{\chi} / 2 a_{\chi}^{\max }\right) \times\left(\mathcal{O}_{\chi} /\left(a_{\chi}^{\min } / 2\right)\right)
$$

as $\mathcal{O}_{\chi}$-modules.

Proof. The hypothesis implies that both $\mathrm{Cl}^{+}(\chi)$ and $\mathrm{Cl}^{-}(\chi)$ are cyclic $\mathcal{O}_{\chi}$-modules. Therefore $\mathrm{Cl}(\chi)$ has $\mathcal{O}_{\chi}$-rank at most 2 . The result now follows from Lemma 1.

We now show that if $l \equiv 3(\bmod 4), \mathrm{Cl}(\chi)$ is a cyclic $\mathcal{O}_{\chi}[P]$-module, and $h_{\chi}^{+} \neq h_{\chi}^{-}$, then the structure of $\mathrm{Cl}(\chi)$ as an $\mathcal{O}_{\chi}$-module, can be determined.

Proposition $7 . \quad$ Let $l \equiv 3(\bmod 4), h_{\chi}^{+}>h_{\chi}^{-}$and suppose that $\mathrm{Cl}(\chi)$ is a cyclic $\mathcal{O}_{\chi}[P]$-module. Then there is an isomorphism of $\mathcal{O}_{\chi}$-modules:

$$
\mathrm{Cl}(\chi) \cong\left(\mathcal{O}_{\chi} / 2 a_{\chi}^{+}\right) \times\left(\mathcal{O}_{\chi} /\left(a_{\chi}^{-} / 2\right)\right)
$$

Proof. Let $x$ be a generator of $\mathrm{Cl}(\chi)$. We consider

$$
x^{2}=x^{1+j} x^{1-j} .
$$

Multiplying by $a_{\chi}^{+} / 2$, we get

$$
x^{a_{\chi}^{+}}=x^{\left(a_{\chi}^{+} / 2\right)(1+j)}
$$

since $a_{\chi}^{+} / 2 \geq a_{\chi}^{-}$kills $\mathrm{Cl}(\chi)^{1-j}$. Since $\mathrm{Cl}(\chi)^{1+j} \cong \mathrm{Cl}^{+}(\chi)$ is cyclic and has exponent $a_{\chi}^{+}$, the right hand side is not trivial. Thus $x$ has order $2 a_{\chi}^{+}$, and we are in the second case of Corollary 3.

Proposition 8. Let $l \equiv 3(\bmod 4), h_{\chi}^{+}<h_{\chi}^{-}$and suppose that $\mathrm{Cl}(\chi)$ is a cyclic $\mathcal{O}_{\chi}[P]$-module. Then we have, as $\mathcal{O}_{\chi}$-modules: 
1. $\mathrm{Cl}(\chi) \cong\left(\mathcal{O}_{\chi} / a_{\chi}^{-}\right) \times\left(\mathcal{O}_{\chi} / a_{\chi}^{+}\right)$if $\mathrm{Cl}(\chi)$ is not cohomologically trivial;

2. $\mathrm{Cl}(\chi) \cong\left(\mathcal{O}_{\chi} / 2 a_{\chi}^{-}\right) \times\left(\mathcal{O}_{\chi} /\left(a_{\chi}^{+} / 2\right)\right)$ if $\mathrm{Cl}(\chi)$ is cohomologically trivial.

Proof. Because of Corollary 3, it is enough to prove that the first condition is verified if and only if $\mathrm{Cl}(\chi)$ is not cohomologically trivial. Suppose that

$$
\mathrm{Cl}(\chi) \cong\left(\mathcal{O}_{\chi} / a_{\chi}^{-}\right) \times\left(\mathcal{O}_{\chi} / a_{\chi}^{+}\right)
$$

as $\mathcal{O}_{\chi}$-modules. The module $\mathrm{Cl}(\chi)$ is killed by $a_{\chi}^{-}$and by $a_{\chi}^{+}(1+j)$. Since $\mathrm{Cl}(\chi)$ is $\mathcal{O}_{\chi}[P]$-cyclic, counting orders we must have an isomorphism of $\mathcal{O}_{\chi}[P]$-modules

$$
\mathrm{Cl}(\chi) \cong \frac{\mathcal{O}_{\chi}[P]}{\left(a_{\chi}^{-}, a_{\chi}^{+}(1+j)\right)} .
$$

It is immediately verified that this is not cohomologically trivial. Now suppose that $\mathrm{Cl}(\chi)$ is not cohomologically trivial. Let $x$ an element of $\mathrm{Cl}(\chi)$. We have

$$
x^{2}=x^{1+j} x^{1-j} .
$$

Since $\widehat{H}^{1}(P, \mathrm{Cl}(\chi))$ is not trivial, we get

$$
\# \mathrm{Cl}(\chi)^{1-j}<\# \operatorname{Ker}(1+j)=\# \mathrm{Cl}^{-}(\chi)=\#\left(\mathcal{O}_{\chi} / a_{\chi}^{-}\right) .
$$

This implies that $a_{\chi}^{-} / 2$ kills $\mathrm{Cl}(\chi)^{1-j}$. But, since $h_{\chi}^{+}<h_{\chi}^{-}$, the number $a_{\chi}^{-} / 2$ kills also $\mathrm{Cl}(\chi)^{1+j}$. If we multiply $(9)$ by $a_{\chi}^{-} / 2$, we get

$$
x^{a_{\chi}^{-}}=1 .
$$

This implies that $\mathrm{Cl}(\chi)$ has exponent $a_{\chi}^{-}$. Therefore

$$
\mathrm{Cl}(\chi) \cong\left(\mathcal{O}_{\chi} / a_{\chi}^{-}\right) \times\left(\mathcal{O}_{\chi} / a_{\chi}^{+}\right)
$$

as $\mathcal{O}_{\chi}$-modules. This completes the proof.

If $h_{\chi}^{+}=h_{\chi}^{-}$, then we do not have a criterion to determine the structure of $\mathrm{Cl}(\chi)$ as an $\mathcal{O}_{\chi}$-module in general. Anyway, the following is true:

Proposition 9. Let $l \equiv 3(\bmod 4), h_{\chi}^{+}=h_{\chi}^{-}$and suppose that $\mathrm{Cl}(\chi)$ is a cyclic $\mathcal{O}_{\chi}[P]$-module. If $\mathrm{Cl}(\chi)$ is not cohomologically trivial, then there is an isomorphism of $\mathcal{O}_{\chi}$-modules:

$$
\mathrm{Cl}(\chi) \cong\left(\mathcal{O}_{\chi} / 2 a_{\chi}^{+}\right) \times\left(\mathcal{O}_{\chi} /\left(a_{\chi}^{+} / 2\right)\right) .
$$


Proof. In our situation we have that $\widehat{H}^{i}(P, \mathrm{Cl}(\chi))$ is isomorphic to $\mathcal{O}_{\chi} / 2$, for all $i \in \mathbb{Z}$. By the properties of Tate cohomology groups

$$
\widehat{H}^{1}(P, \mathrm{Cl}(\chi)) \cong{ }_{N} \mathrm{Cl}(\chi) / \mathrm{Cl}(\chi)^{1-j}
$$

where ${ }_{N} \mathrm{Cl}(\chi)$ denotes the kernel of the norm map

$$
\mathrm{Cl}(\chi) \rightarrow \mathrm{Cl}(\chi): x \rightarrow x^{1+j}
$$

We have that $\#\left({ }_{N} \mathrm{Cl}(\chi)\right)=h_{\chi}^{-}$. From this we get easily that

$$
\# \mathrm{Cl}(\chi)^{1-j}=\#\left(\mathcal{O}_{\chi} /\left(a_{\chi}^{-} / 2\right)\right) \text {. }
$$

In particular, $a_{\chi}^{-} / 2$ kills $\mathrm{Cl}(\chi)^{1-j}$. On the other hand, since $\mathrm{Cl}^{+}(\chi) \cong$ $\mathrm{Cl}(\chi)^{1+j}$ is a cyclic $\mathcal{O}_{\chi}$-module, $\mathrm{Cl}(\chi)^{1+j} \cong \mathcal{O}_{\chi} / a_{\chi}^{+}$. Therefore $a_{\chi}^{+}$is the exponent of $\mathrm{Cl}(\chi)^{1+j}$. Now let $x$ be a generator of $\mathrm{Cl}(\chi)$. We have

$$
x^{2}=x^{1+j} x^{1-j} .
$$

It is now easy to see that $x$ has order $2 a_{\chi}^{+}$. Thus we are in the second case of Corollary 3.

\subsection{The general case}

In this subsection we give some results which are a generalization of the ones in the previous subsection. If $A$ is any finite abelian group, we denote by $\operatorname{Exp}(A)$ its exponent.

Proposition 10. Suppose that $\mathrm{Cl}(\chi)$ is a cyclic $\mathcal{O}_{\chi}[P]$-module. Let $Q$ be the cyclic group of order 2 generated by $j$. Suppose that

$$
\# \widehat{H}^{0}(Q, \mathrm{Cl}(\chi))=h_{\chi}^{-}
$$

Then

$$
\#\left(\mathrm{Cl}(\chi) / \mathrm{Cl}(\chi)^{2}\right)=h_{\chi}^{-}
$$

and $\operatorname{Exp}(\mathrm{Cl}(\chi))=2 \operatorname{Exp}\left(\mathrm{Cl}(\chi)^{1+j}\right)$.

Proof. We have an isomorphism

$$
\widehat{H}^{0}(Q, \mathrm{Cl}(\chi)) \cong \mathrm{Cl}(\chi)^{Q} / \mathrm{Cl}(\chi)^{1+j} .
$$

This implies that our assumption on the order of the cohomology group is equivalent to the equality $\mathrm{Cl}(\chi)^{Q}=\mathrm{Cl}(\chi)$. Since in this case $j$ acts as 
identity on $\mathrm{Cl}(\chi)$, we get $\mathrm{Cl}(\chi)^{2}=\mathrm{Cl}(\chi)^{1+j}$. The proof of the first part of the proposition follows substituting these relations in our hypothesis. Let now $x$ be a generator of $\mathrm{Cl}(\chi)$. The element $x^{2}=x^{1+j}$ is a generator of $\mathrm{Cl}(\chi)^{1+j} \cong \mathrm{Cl}^{+}(\chi)$. Thus $2 \operatorname{Exp}\left(\mathrm{Cl}(\chi)^{1+j}\right)=\operatorname{Exp}(\mathrm{Cl}(\chi))$, as we wanted to prove.

Proposition 11. Suppose that $\mathrm{Cl}(\chi)$ is a cyclic $\mathcal{O}_{\chi}[P]$-module. Suppose that $\operatorname{Exp}\left(\mathrm{Cl}^{+}(\chi)\right)>\operatorname{Exp}\left(\mathrm{Cl}^{-}(\chi)\right)$. Then $\operatorname{Exp}(\mathrm{Cl}(\chi))=2 \operatorname{Exp}\left(\mathrm{Cl}^{+}(\chi)\right)$.

Proof. We first want to show that $\# \mathrm{Cl}(\chi)^{1-j} \leq h_{\chi}^{-}$. This is true because

$$
\mathrm{Cl}(\chi)^{1-j} \subset \operatorname{Ker}\left(1+j: \mathrm{Cl}(\chi) \rightarrow \mathrm{Cl}(\chi)^{1+j}\right)
$$

and the right hand side has order $h_{\chi}^{-}$. Both $\mathrm{Cl}(\chi)^{1-j}$ and $\mathrm{Cl}^{-}(\chi)$ are cyclic modules over the discrete valuation ring $\mathcal{O}_{\chi}[P] /(1+j)$. Since $\mathrm{Cl}(\chi)^{1-j}$ has order less or equal than $\# \mathrm{Cl}^{-}(\chi)=h_{\chi}^{-}$, it follows that $\mathrm{Cl}(\chi)^{1-j}$ is isomorphic to a quotient of $\mathrm{Cl}^{-}(\chi)$. This implies that $\operatorname{Exp}\left(\mathrm{Cl}(\chi)^{1-j}\right) \leq$ $\operatorname{Exp}\left(\mathrm{Cl}^{-}(\chi)\right)<\operatorname{Exp}\left(\mathrm{Cl}^{+}(\chi)\right)$. Let $x$ be a generator of $\mathrm{Cl}(\chi)$. We consider the identity

$$
x^{2}=x^{1+j} x^{1-j} .
$$

It is easy to see that the order of the right hand side is $\operatorname{Exp}\left(\mathrm{Cl}(\chi)^{1+j}\right)=$ $\operatorname{Exp}\left(\mathrm{Cl}^{+}(\chi)\right)$. Looking at the left hand side, we get that the order of $x$ is $2 \operatorname{Exp}\left(\mathrm{Cl}^{+}(\chi)\right)$. This completes the proof.

The following proposition deals with a very ad hoc situation. It will enable us to determine the exponent of $\mathrm{Cl}(\chi)$ in the cases $l=397$ and $l=9421$.

Proposition $12 . \quad$ Let $l \equiv 5(\bmod 8)$. Suppose that $\# \mathrm{Cl}^{-}(\chi)=\# \mathcal{O}_{\chi} / 8$, and $\# \mathrm{Cl}^{+}(\chi)=\# \mathcal{O}_{\chi} / 2$. If $\mathrm{Cl}(\chi)$ is a cyclic $\mathcal{O}_{\chi}[P]$-module, then it has exponent equal to 4 .

Proof. Combining Theorem 1 of [4] and Proposition 7 (iii) of [4] we get that

$$
\widehat{H}^{0}(Q, \mathrm{Cl}(\chi)) \cong\left(\mathcal{O}_{F,+}^{*} /\left(\mathcal{O}_{F}^{*}\right)^{2}\right)(\chi)
$$

where $F=\mathbb{Q}\left(\zeta_{l}+\zeta_{l}^{-1}\right)$ and $Q$ is the subgroup of $P$ of order 2 generated by $j$. We have $e=2$ and $\mathrm{Cl}^{+}(\chi)=\mathrm{Cl}_{1}(\chi)$. By contradiction, suppose that $\# \mathrm{Cl}_{1}^{\infty}(\chi)=\# \mathrm{Cl}_{1}(\chi)=\#\left(\mathcal{O}_{\chi} / 2\right)$. Since by Proposition 5 the group $\mathrm{Cl}_{0}^{\infty}(\chi)$ is not trivial, we get that the surjective map $\mathrm{Cl}_{1}^{\infty}(\chi) \rightarrow \mathrm{Cl}_{0}^{\infty}(\chi)$ induced 
by the norm is actually an isomorphism. Let $\sigma$ be a generator of $P$. From Equation (1) of Proposition 1 we obtain

$$
\mathrm{Cl}(\chi)^{1-\sigma}=\mathrm{Cl}(\chi)^{1-\sigma^{2}}=\left(\mathrm{Cl}(\chi)^{1-\sigma}\right)^{1+\sigma} .
$$

Since the element $1+\sigma$ is contained in the maximal ideal of the local ring $\mathcal{O}_{\chi}[P]$, Nakayama's lemma gives $\mathrm{Cl}(\chi)^{1-\sigma} \cong 0$. Proposition 1 then implies that $\mathrm{Cl}(\chi) \cong \mathrm{Cl}_{1}(\chi) \cong \mathrm{Cl}^{+}(\chi)$, which is absurd, because $\mathrm{Cl}^{-}(\chi)$ is not trivial. Therefore $\# \mathrm{Cl}_{1}^{\infty}(\chi)>\# \mathrm{Cl}_{1}(\chi)$ and $\left(\mathcal{O}_{F,+}^{*} /\left(\mathcal{O}_{F}^{*}\right)^{2}\right)(\chi)$ is not trivial. This implies that the Tate cohomology group $\widehat{H}^{1}(Q, \mathrm{Cl}(\chi))$ is not trivial. Therefore $\mathrm{Cl}(\chi)^{1-j}$ has order strictly smaller than $h_{\chi}^{-}$, hence $\# \mathrm{Cl}(\chi)^{1-j} \leq$ $\#\left(\mathcal{O}_{\chi} / 4\right)$. The group $\mathrm{Cl}(\chi)^{1-j}$ is a cyclic module over $\mathcal{O}_{\chi}[P] /(1+j)$. By Proposition 2 we get that $\mathrm{Cl}(\chi)^{1-j}$ can have at most exponent equal to 2 . Let $x$ be a generator of $\mathrm{Cl}(\chi)$. From the usual identity

$$
x^{2}=x^{1+j} x^{1-j}
$$

we see that $\mathrm{Cl}(\chi)$ has at most exponent 4 . If we show that $\mathrm{Cl}^{-}(\chi)$ has exponent 4 , then the proof is complete. But again $\mathrm{Cl}^{-}(\chi)$ is a cyclic $\mathcal{O}_{\chi}[P] /(1+$ $j)$-module of order $\# \mathcal{O}_{\chi} / 8$. By Proposition 2 such a module has exponent 4.

\section{$\S 5$. Tables}

Let $l$ be a prime number and let $\chi$ be a 2-adic character as in the previous sections. The theory developed allows us to determine much information about $\mathrm{Cl}(\chi)$, and sometimes the whole structure as an $\mathcal{O}_{\chi}$-module. In this section we present a table containing our numerical results. If $\mathrm{Cl}^{+}(\chi)$ is trivial then $\mathrm{Cl}(\chi) \cong \mathrm{Cl}^{-}(\chi)$. In this case, using Propositions 6 and 2 it is easy to determine the whole $\mathcal{O}_{\chi}[P]$-structure of $\mathrm{Cl}^{-}(\chi)$ from the knowledge of its order. The table has an entry for each prime number $l<10000 \mathrm{such}$ that $\mathrm{Cl}^{+}(\chi)$ is not trivial. For each $l$ we determine various quantities. The number $d$ denotes the degree of the field $K_{e} \subset \mathbb{Q}\left(\zeta_{l}\right)$ fixed by $\operatorname{Ker}(\chi)$. The numbers $h_{\chi}^{+}$and $h_{\chi}^{-}$denote the order of $\mathrm{Cl}^{+}(\chi)$ and of $\mathrm{Cl}^{-}(\chi)$ respectively. They have been computed in [4]. The column \# $\widehat{H}^{0}$ contains the order of the Tate cohomology group $\widehat{H}^{0}(Q, \mathrm{Cl}(\chi))$, where $Q$ is the group of order 2 generated by complex conjugation. This quantity can be easily computed using the table and Theorem 1 of [4]. The other entries contain the structure of the groups $\mathrm{Cl}^{+}(\chi), \mathrm{Cl}^{-}(\chi)$ and $\mathrm{Cl}(\chi)$ as $\mathcal{O}_{\chi}$-modules. In the table 
we write $n$ for $\mathcal{O}_{\chi} / n$. Observe that as an abelian group we have

$$
\mathcal{O}_{\chi} / 2^{k} \cong\left(\mathbb{Z} / 2^{k} \mathbb{Z}\right)^{f_{\chi}}
$$

where $f_{\chi}=\left[\mathbb{Z}_{2}(\chi): \mathbb{Z}_{2}\right]$ is the multiplicative order of 2 in $(\mathbb{Z} / \operatorname{ord}(\chi))^{*}$. We are not able to determine these structures in all cases. Therefore some entries are left blank. The structure of $\mathrm{Cl}^{+}(\chi)$ and of $\mathrm{Cl}^{-}(\chi)$ are computed in all cases when $\mathrm{Cl}(\chi)$ is a cyclic $\mathcal{O}_{\chi}[P]$-module. By Theorem 2, this happens when $l \neq 7687$. The groups $\mathrm{Cl}^{+}(\chi)$ have been computed from the ideals $J^{+}(\chi)$ which can be found in the table of [17] (see the remarks in the introduction). The groups $\mathrm{Cl}^{-}(\chi)$ can be computed using Proposition 2 . The groups $\mathrm{Cl}(\chi)$ have been computed in some cases, using the results mentioned in the column labelled as "notes". It turns out that for each $l<10000$ there is at most one character $\chi$ such that $\mathrm{Cl}^{+}(\chi)$ is not trivial, except for $l=7841$. For $l=7841$ there are exactly two characters with this property; in this case the table has two entries corresponding to each character. In the two cases $l=397$ and $l=9421$, we are able to determine only the exponent of the class group $\mathrm{Cl}(\chi)$.

\begin{tabular}{|r|r|r|r|r|r|r|r|r|}
\hline$l$ & $d$ & $h_{\chi}^{+}$ & $h_{\chi}^{-}$ & $\# \widehat{H}^{0}$ & $\mathrm{Cl}^{+}(\chi)$ & $\mathrm{Cl}^{-}(\chi)$ & $\mathrm{Cl}(\chi)$ & notes \\
\hline 163 & 6 & $2^{2}$ & $2^{2}$ & 1 & 2 & 2 & & \\
277 & 12 & $2^{2}$ & $2^{4}$ & $2^{2}$ & 2 & 2,2 & & \\
349 & 12 & $2^{4}$ & $2^{4}$ & 1 & 2,2 & 2,2 & & \\
397 & 12 & $2^{2}$ & $2^{6}$ & $2^{2}$ & 2 & 2,4 & $\mathrm{Exp}=4$ & prop. 12 \\
491 & 14 & $2^{3}$ & $2^{3}$ & 1 & 2 & 2 & & \\
547 & 6 & $2^{2}$ & $2^{2}$ & 1 & 2 & 2 & & \\
607 & 6 & $2^{2}$ & $2^{4}$ & 1 & 2 & 4 & 8 & prop. 8 \\
709 & 12 & $2^{4}$ & $2^{4}$ & 1 & 2,2 & 2,2 & & \\
827 & 14 & $2^{3}$ & $2^{3}$ & 1 & 2 & 2 & & \\
853 & 12 & $2^{2}$ & $2^{2}$ & $2^{2}$ & 2 & 2 & 4 & th. 1 \\
937 & 24 & $2^{4}$ & $2^{2}$ & $2^{2}$ & 4 & 2 & 8 & th. 1 \\
941 & 20 & $2^{4}$ & $2^{8}$ & $2^{4}$ & 2 & 2,2 & & \\
1009 & 48 & $2^{2}$ & $2^{2}$ & $2^{2}$ & 2 & 2 & 4 & th. 1 \\
1399 & 6 & $2^{2}$ & $2^{4}$ & 1 & 2 & 4 & 8 & prop. 8 \\
1699 & 6 & $2^{2}$ & $2^{2}$ & $2^{2}$ & 2 & 2 & 4 & prop. 9 \\
1777 & 48 & $2^{4}$ & $2^{4}$ & $2^{4}$ & 4 & 2,2 & 2,8 & prop. 11 \\
1789 & 12 & $2^{2}$ & $2^{4}$ & $2^{2}$ & 2 & 2,2 & & \\
1879 & 6 & $2^{2}$ & $2^{2}$ & 1 & 2 & 2 & & \\
1951 & 6 & $2^{2}$ & $2^{2}$ & 1 & 2 & 2 & & \\
\hline
\end{tabular}




\begin{tabular}{|c|c|c|c|c|c|c|c|c|}
\hline$l$ & $d$ & $h_{\chi}^{+}$ & $h_{\chi}^{-}$ & $\# \widehat{H}^{0}$ & $\mathrm{Cl}^{+}(\chi)$ & $\mathrm{Cl}^{-}(\chi)$ & $\mathrm{Cl}(\chi)$ & notes \\
\hline 2131 & 6 & $2^{2}$ & $2^{2}$ & 1 & 2 & 2 & & \\
\hline 2161 & 80 & $2^{4}$ & $2^{4}$ & $2^{4}$ & 2 & 2 & 4 & th. 1 \\
\hline 2311 & 6 & $2^{2}$ & $2^{2}$ & 1 & 2 & 2 & & \\
\hline 2689 & 384 & $2^{2}$ & $2^{2}$ & $2^{2}$ & 2 & 2 & 4 & th. 1 \\
\hline 2797 & 12 & $2^{2}$ & $2^{4}$ & $2^{2}$ & 2 & 2,2 & & \\
\hline 2803 & 6 & $2^{2}$ & $2^{2}$ & 1 & 2 & 2 & & \\
\hline 2927 & 14 & $2^{3}$ & $2^{3}$ & 1 & 2 & 2 & & \\
\hline 3037 & 12 & $2^{2}$ & $2^{4}$ & $2^{2}$ & 2 & 2,2 & & \\
\hline 3271 & 6 & $2^{2}$ & $2^{2}$ & 1 & 2 & 2 & & \\
\hline 3301 & 20 & $2^{4}$ & $2^{4}$ & $2^{4}$ & 2 & 2 & 4 & th. 1 \\
\hline 3517 & 12 & $2^{2}$ & $2^{2}$ & $2^{2}$ & 2 & 2 & 4 & th. 1 \\
\hline 3727 & 6 & $2^{2}$ & $2^{2}$ & 1 & 2 & 2 & & \\
\hline 3931 & 10 & $2^{8}$ & $2^{4}$ & 1 & 4 & 2 & 8 & prop. 7 \\
\hline 4099 & 6 & $2^{2}$ & $2^{2}$ & 1 & 2 & 2 & & \\
\hline 4219 & 6 & $2^{2}$ & $2^{2}$ & 1 & 2 & 2 & & \\
\hline 4261 & 12 & $2^{4}$ & $2^{4}$ & 1 & 2,2 & 2,2 & & \\
\hline 4297 & 24 & $2^{8}$ & $2^{4}$ & $2^{4}$ & 2,8 & 2,2 & 4,16 & prop. 10 \\
\hline 4327 & 14 & $2^{3}$ & $2^{3}$ & 1 & 2 & 2 & & \\
\hline 4357 & 12 & $2^{4}$ & $2^{4}$ & $2^{2}$ & 4 & 2,2 & 2,8 & prop. 11 \\
\hline 4561 & 48 & $2^{4}$ & $2^{4}$ & $2^{4}$ & 2,2 & 2,2 & 4,4 & prop. 10 \\
\hline 4567 & 6 & $2^{2}$ & $2^{2}$ & 1 & 2 & 2 & & \\
\hline 4639 & 6 & $2^{2}$ & $2^{2}$ & 1 & 2 & 2 & & \\
\hline 4789 & 12 & $2^{2}$ & $2^{4}$ & $2^{2}$ & 2 & 2,2 & & \\
\hline 4801 & 192 & $2^{2}$ & $2^{4}$ & $2^{4}$ & 2 & 2,2 & 2,4 & prop. 10 \\
\hline 5197 & 12 & $2^{2}$ & $2^{4}$ & $2^{2}$ & 2 & 2,2 & & \\
\hline 5479 & 6 & $2^{2}$ & $2^{2}$ & 1 & 2 & 2 & & \\
\hline 5531 & 14 & $2^{3}$ & $2^{3}$ & 1 & 2 & 2 & & \\
\hline 5659 & 6 & $2^{2}$ & $2^{2}$ & 1 & 2 & 2 & & \\
\hline 5779 & 6 & $2^{2}$ & $2^{2}$ & 1 & 2 & 2 & & \\
\hline 5953 & 192 & $2^{2}$ & $2^{2}$ & $2^{2}$ & 2 & 2 & 4 & th. 1 \\
\hline 6037 & 12 & $2^{2}$ & $2^{2}$ & $2^{2}$ & 2 & 2 & 4 & th. 1 \\
\hline 6079 & 6 & $2^{2}$ & $2^{2}$ & 1 & 2 & 2 & & \\
\hline 6163 & 6 & $2^{2}$ & $2^{6}$ & 1 & 2 & 8 & 16 & prop. 8 \\
\hline 6247 & 6 & $2^{4}$ & $2^{6}$ & 1 & 4 & 8 & 2,16 & prop. 8 \\
\hline 6301 & 28 & $2^{3}$ & $2^{3}$ & $2^{3}$ & 2 & 2 & 4 & th. 1 \\
\hline 6553 & 24 & $2^{2}$ & $2^{2}$ & $2^{2}$ & 2 & 2 & 4 & th. 1 \\
\hline
\end{tabular}




\begin{tabular}{|r|r|r|r|r|r|r|r|r|}
\hline$l$ & $d$ & $h_{\chi}^{+}$ & $h_{\chi}^{-}$ & $\# \widehat{H}^{0}$ & $\mathrm{Cl}^{+}(\chi)$ & $\mathrm{Cl}^{-}(\chi)$ & $\mathrm{Cl}(\chi)$ & notes \\
\hline 6637 & 12 & $2^{2}$ & $2^{2}$ & $2^{2}$ & 2 & 2 & 4 & th. 1 \\
6709 & 12 & $2^{2}$ & $2^{2}$ & $2^{2}$ & 2 & 2 & 4 & th. 1 \\
6833 & 112 & $2^{3}$ & $2^{3}$ & $2^{3}$ & 2 & 2 & 4 & th. 1 \\
7027 & 6 & $2^{2}$ & $2^{2}$ & $2^{2}$ & 2 & 2 & 4 & prop. 9 \\
7297 & 384 & $2^{2}$ & $2^{4}$ & $2^{4}$ & 2 & 2,2 & 2,4 & prop. 10 \\
7489 & 192 & $2^{6}$ & $2^{2}$ & $2^{2}$ & 8 & 2 & 16 & th. 1 \\
7589 & 28 & $2^{3}$ & $2^{3}$ & $2^{3}$ & 2 & 2 & 4 & th. 1 \\
7639 & 6 & $2^{2}$ & $2^{4}$ & $2^{2}$ & 2 & 4 & 2,4 & prop. 8 \\
7687 & 6 & $2^{4}$ & $2^{4}$ & 1 & & & & \\
7841 & 224 & $2^{3}$ & $2^{3}$ & $2^{3}$ & 2 & 2 & 4 & th. 1 \\
& 224 & $2^{3}$ & $2^{3}$ & $2^{3}$ & 2 & 2 & 4 & th. 1 \\
7867 & 6 & $2^{2}$ & $2^{2}$ & 1 & 2 & 2 & & \\
7879 & 6 & $2^{2}$ & $2^{2}$ & 1 & 2 & 2 & & \\
8011 & 6 & $2^{2}$ & $2^{2}$ & 1 & 2 & 2 & & \\
8191 & 6 & $2^{2}$ & $2^{4}$ & $2^{2}$ & 2 & 4 & 2,4 & prop. 8 \\
8209 & 48 & $2^{2}$ & $2^{2}$ & $2^{2}$ & 2 & 2 & 4 & th. 1 \\
8629 & 12 & $2^{2}$ & $2^{2}$ & $2^{2}$ & 2 & 2 & 4 & th. 1 \\
8647 & 6 & $2^{2}$ & $2^{2}$ & 1 & 2 & 2 & & \\
8731 & 6 & $2^{2}$ & $2^{2}$ & 1 & 2 & 2 & & \\
8831 & 10 & $2^{4}$ & $2^{4}$ & 1 & 2 & 2 & & \\
8887 & 6 & $2^{2}$ & $2^{2}$ & 1 & 2 & 2 & & \\
9109 & 12 & $2^{4}$ & $2^{4}$ & $2^{2}$ & 4 & 2,2 & 2,8 & prop. 11 \\
9283 & 6 & $2^{2}$ & $2^{2}$ & $2^{2}$ & 2 & 2 & 4 & prop. 9 \\
9319 & 6 & $2^{2}$ & $2^{2}$ & 1 & 2 & 2 & & \\
9337 & 24 & $2^{6}$ & $2^{2}$ & 1 & 8 & 2 & 16 & th. 1 \\
9391 & 6 & $2^{2}$ & $2^{2}$ & 1 & 2 & 2 & & \\
9421 & 12 & $2^{2}$ & $2^{6}$ & $2^{2}$ & 2 & 2,4 & $\mathrm{Exp}=4$ & prop. 12 \\
9601 & 384 & $2^{4}$ & $2^{2}$ & $2^{2}$ & 4 & 2 & 8 & th. 1 \\
9649 & 48 & $2^{2}$ & $2^{4}$ & $2^{4}$ & 2 & 2,2 & 2,4 & prop. 10 \\
9721 & 24 & $2^{2}$ & $2^{2}$ & $2^{2}$ & 2 & 2 & 4 & th. 1 \\
\hline
\end{tabular}

\section{REFERENCES}

[1] T. Berthier, Générateurs et structure du groupe des classes d'idéaux des corps de nombres abéliens, thése, Univ. de Franche-Comté, 1994.

[2] P. E. Conner and J. Hurrelbrink, Class number parity, Series in pure mathematics 8, World Scientific, Singapore 1988. 
[3] P. Cornacchia, The parity of the class number of the cyclotomic fields of prime conductor, Proceedings of the A.M.S., 125, n.11 (1997), 3163-3168.

[4] _ Anderson's module for cyclotomic fields of prime conductor, J. Number Theory, 67, 2 (1997), 252-276.

[5] P. Cornacchia and C. Greither, Fitting Ideals of Class Groups of Real Fields with Prime Power Conductor, J. Number Theory, 73 (1998), 459-471.

[6] G. Gras, Nombre de $\phi$-classes invariantes, application aux classes des corps abéliens, Bull. Soc. Math. de France, 106 (1978), 337-364.

[7] G. Gras and T. Berthier, Sur la structure des groupes de classes relatives, avec un appendice d'exemples numériques, Ann. Inst. Fourier, Grenoble, 43, 1 (1993), 1-20.

[8] M. N. Gras, Méthodes et algorithmes pour le calcul numérique du nombre de classes et des unités des extensions cubiques cycliques de $\mathbb{Q}$, J. Reine Angew. Math., 277 (1975), 89-116.

[9] C. Greither, Class groups of abelian fields, and the main conjecture, Ann. Inst. Fourier, Grenoble, 42, 3 (1992), 449-499.

[10] G. Guerry, Sur la 2-composante du groupe des classes de certaines extensions cycliques de degré $2^{N}$, J. Number Theory, 53 (1995), 159-172.

[11] S. Lang, Cyclotomic fields I and II, combined 2nd edition, Graduate Texts in Math., 121, Springer Verlag, New York 1990.

[12] B. Oriat, Relation entre les 2-groupes des classes d'idéaux au sens ordinaire et restreint de certains corps de nombres, Bull. Soc. math. France, 104 (1976), 301-307.

[13] K. Rubin, Global units and ideal class groups, Invent. Math., 89 (1987), 511-526.

[14] The Main Conjecture, Appendix to [11].

[15] R. Schoof, Minus class groups of the fields of the l-th roots of unity, Math. of Comp., 67 (1998), 1225-1245.

[16] — The structure of the minus class groups of abelian number fields, Séminaire de Théorie des Nombres, Paris 1988-89, 185-204.

[17] Class numbers of real cyclotomic fields of prime conductor, preprint.

[18] L.C. Washington, Introduction to cyclotomic fields, 2nd edition, Graduate Texts in Math. 83, Springer Verlag, New York 1997.

Corso XXV Aprile 60

14100 Asti

Italy

cornac@dm.unipi.it 